\title{
Données et démographie: informatif et passionnant (1)
}

La FMH a une longue tradition dans le traitement des données relatives à la démographie médicale. Celles-ci sont utiles aux sociétés de discipline médicale, aux sociétés cantonales de médecine, aux médecins et aux différentes organisations pour former leur opinion politique, forger leurs argumentations, planifier leurs besoins et réaliser leurs études. Le Département Données, Démographie et Qualité de la FMH propose jusqu'à la fin de l'année une série d'articles sur les sujets les plus demandés. Pour toute question ou évaluation plus détaillée, n'hésitez pas à vous adresser au département DDQ: ddq@fmh.ch / 0313591111.

Esther Kraft, Martina Hersperger

Correspondance:

Esther Kraft

FMH, Département DDQ

Elfenstrasse 18

CH-3000 Berne 15

Tél. 0313591111

Fax 0313591112

esther.kraft@fmh.ch

Figure 1

Evolution du nombre de médecins en exercice

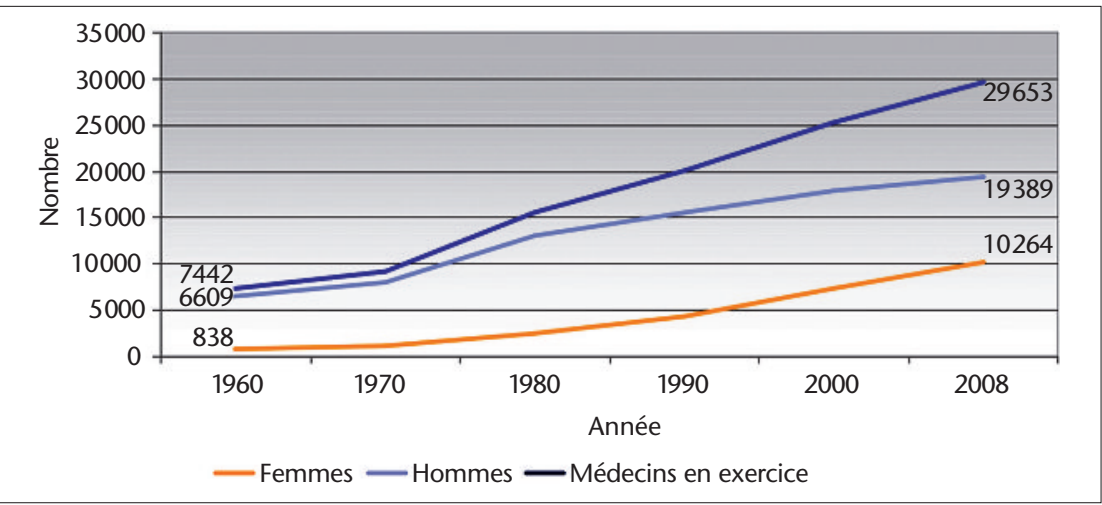

L'évolution du corps médical est recensée de manière chiffrée depuis les années 30 et cela fait 70 ans que la FMH publie régulièrement sa statistique médicale. L'histoire, les caractéristiques principales et les événements qui l'ont marquée, de même que la répartition géographique, la féminisation de la profession, la formation prégraduée et postgraduée, l'évolution du cursus des médecins, etc. feront l'objet d'une série d'articles qui paraîtront dans le BMS jusqu'à la fin de

L'article introductif offre un aperçu de l'évolution qui a marqué le corps médical depuis les années 60 en accordant une attention particulière à l'évolution chiffrée, à la densité médicale et à la formation des futurs médecins.

En 1960, 7442 médecins exerçaient en Suisse et la part des femmes était de 11,9\% [1]. En 2008, ce chiffre est passé à 29653 [2], dont 34,4\% de femmes. Le nombre de médecins en exercice a donc fait un bon de $400 \%$ en 48 ans (cf. graphique 1). Si on le compare

\section{8 en chiffres}

- A la fin de l'année, env. $\mathbf{3 0 0 0 0}$ médecins exerçaient en Suisse. l'année.

- Parmi eux, 34,4\% sont des femmes.

- Plus de $60 \%$ des étudiants en médecine sont des femmes.

- Depuis 1960, le nombre de médecins a augmenté de $400 \%$.

à l'augmentation de la population suisse $(+43,7 \%$ depuis 1960 [3]), on constate que la densité médicale (c'est-à-dire le nombre d'habitants par médecin) a augmenté.

On compte actuellement 1 médecin pour 260 habitants, mais les disparités cantonales sont importantes. Les cantons de Zurich, Berne, Bâle-Ville, Vaud et Genève ont la plus forte densité médicale, tant dans le secteur ambulatoire qu'hospitalier. C'était déjà le cas dans les années 60, de même pour le canton d'Appenzell Rhodes-Intérieures qui avait déjà la plus faible densité médicale à l'époque.

Pour pouvoir analyser l'évolution socio-démographique et les spécificités des médecins, il importe de disposer d'informations sur les étudiants en médecine humaine. Celles-ci permettront d'établir des pronostics sur les futurs effectifs médicaux. Depuis le début du $\mathrm{XX}^{\mathrm{e}}$ siècle, l'intérêt pour la nationalité et le sexe des étudiants en médecine augmente. A l'époque, le nombre d'étudiants suisses et étrangers était équilibré. Pendant les deux guerres mondiales, la part d'étudiants étrangers fut inférieure à $10 \%$. Après la guerre et jusque dans les années 60 , ce chiffre est remonté à 39,9\%. Actuellement, $12,2 \%$ des étudiants en médecine sont de nationalité étrangère.

La question de la féminisation de la profession avait déjà été soulevée par la FMH en 1935, car le nombre de femmes parmi les étudiants était en constante aug- 
mentation. Jusqu'en 1960, $13 \%$ des étudiants étaient des femmes [4] et en l'an 2000, ce taux a pour la première fois dépassé celui des hommes (cf. graphique 2). A l'heure actuelle, 7606 étudiants sont immatriculés dans les facultés suisses de médecine et la part des femmes équivaut à 58,8\%.

Cette évolution s'est également traduite dans les diplômes en médecine humaine, ce qui a fait qu'en 2002 et pour la première fois, plus de femmes que d'hommes ont obtenu leur diplôme en médecine (cf. graphique 3). Les années qui suivirent, il y eut à nouveau plus d'hommes que de femmes. Depuis 2004, le nombre de femmes qui terminent avec succès leurs

Figure 2

Evolution du nombre d'étudiants en médecine humaine.

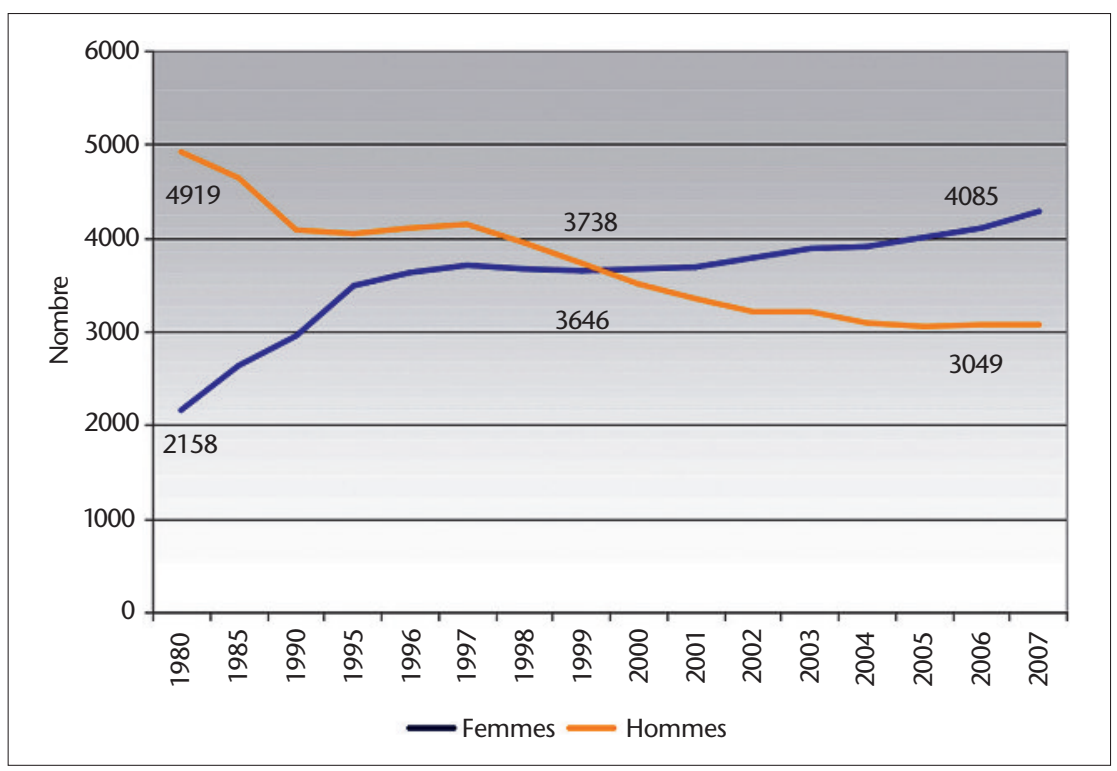

Figure 3

Evolution du nombre de diplômes en médecine humaine.

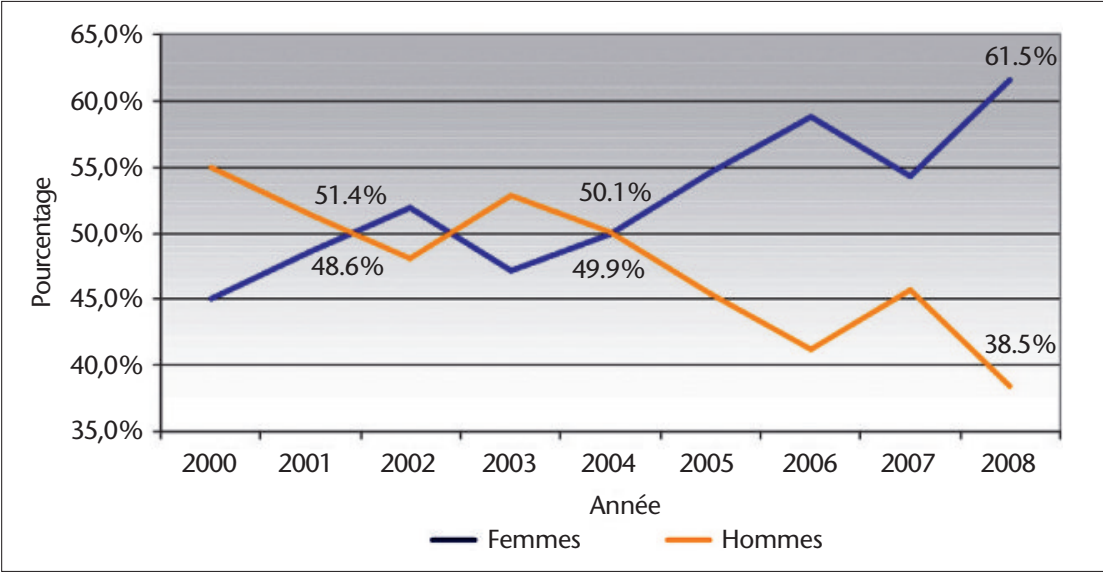

études de médecine est de plus de $50 \%$ et ce taux a même atteint $60 \%$ l'année dernière.

Les prochains articles traiteront en détail des sujets mentionnés dans l'introduction. Le prochain article consacré à l'histoire du corps médical paraîtra le 7 octobre 2009 dans le BMS nº 41 .

\section{Références}

1 Statistique médicale de 1960; Bull Méd Suisses. 1960;(36/37).

2 Kraft E.Statistique médicale de la FMH 2008, nouveaux chiffres - nouveau contenu - nouvelle présentation. Bull Méd Suisses. 2009;90(12):455-61.

3 Office fédéral de la statistique (OFS), Neuchâtel: http://www.bfs.admin.ch/bfs/portal/fr/index/ themen/01/02/blank/data/01.html; 20.7.2009.

4 Wildeisen, E. Zur Frage der Überfüllung des Ärztestandes in der Schweiz. Bull Méd Suisses. 1936;17(32):332-9.

\section{La statistique médicale de la FMH: données de qualité grâce à vous!}

Afin que la FMH puisse répondre aussi à l'avenir aux questions relatives à la démographie actuelle des médecins, la statistique révisée contient depuis 2008 également des précisions sur l'activité menée par les médecins.

En déclarant vos données, vous fournissez une contribution essentielle à une base de discussion et d'argumentation solide pour la régulation du corps médical.

Le nouveau questionnaire figure sur le portail internet www.myfmh.ch sous «Activité professionnelle, questions pour la statistique médicale de la $\mathrm{FMH}$ ». Vous trouverez la statistique médicale de la FMH et les évaluations détaillées sous www.fmh.ch. Celle-ci est également présentée de manière compacte et claire dans notre dépliant disponible gratuitement (jusqu'à épuisement du stock) à l'adresse suivante: ddq@fmh.ch

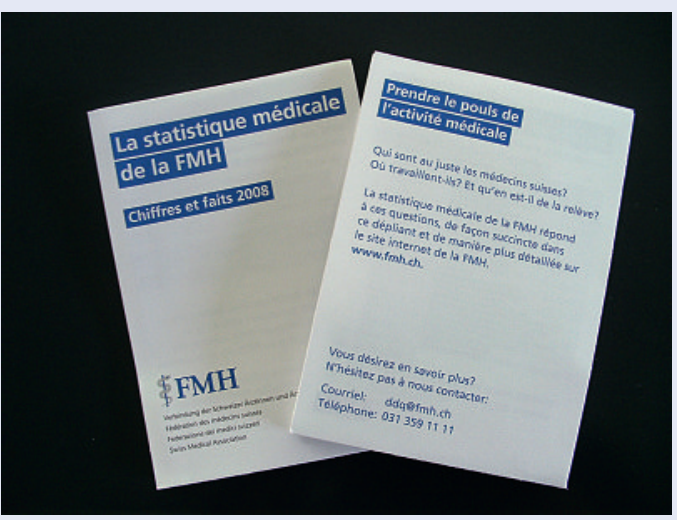

